\title{
VALIDAÇÃO EM MÉTODOS CROMATOGRÁFICOS E ELETROFORÉTICOS
}

Marcelo Ribani

Instituto de Tecnologia do Paraná, Rua Prof. Algacir M. Mader, 3775, 81350-010 Curitiba - PR

Carla Beatriz Grespan Bottoli, Carol H. Collins e Isabel Cristina Sales Fontes Jardim*

Instituto de Química, Universidade Estadual de Campinas, CP 6154, 13084-971 Campinas - SP

Lúcio Flávio Costa Melo

Faculdade de Engenharia Química, Universidade Estadual de Campinas, CP 6066, 13081-971 Campinas - SP

Recebido em 11/6/03; aceito em 2/12/03; publicado na web em 17/6/04

\begin{abstract}
VALIDATION FOR CHROMATOGRAPHIC AND ELECTROPHORETIC METHODS. The validation of an analytical method is fundamental to implementing a quality control system in any analytical laboratory. As the separation techniques, GC, HPLC and $\mathrm{CE}$, are often the principal tools used in such determinations, procedure validation is a necessity. The objective of this review is to describe the main aspects of validation in chromatographic and electrophoretic analysis, showing, in a general way, the similarities and differences between the guidelines established by the different Brazilian and international regulatory agencies.
\end{abstract}

Keywords: validation; legislation; separation methods.

\section{INTRODUÇÃO}

A necessidade de se mostrar a qualidade de medições químicas, através de sua comparabilidade, rastreabilidade e confiabilidade, está sendo cada vez mais reconhecida e exigida. Dados analíticos não confiáveis podem conduzir a decisões desastrosas e a prejuízos financeiros irreparáveis. Para garantir que um novo método analítico gere informações confiáveis e interpretáveis sobre a amostra, ele deve sofrer uma avaliação denominada validação. A validação de um método é um processo contínuo que começa no planejamento da estratégia analítica e continua ao longo de todo o seu desenvolvimento e transferência. Para registro de novos produtos, todos os órgãos reguladores do Brasil e de outros países exigem a validação de metodologia analítica e, para isso, a maioria deles tem estabelecido documentos oficiais que são diretrizes a serem adotadas no processo de validação ${ }^{1-15}$. Um processo de validação bem definido e documentado oferece às agências reguladoras evidências objetivas de que os métodos e os sistemas são adequados para o uso desejado.

As técnicas de separação, tais como cromatografia gasosa (GC), cromatografia líquida de alta eficiência (HPLC) e eletroforese capilar (CE), vêm se destacando na química analítica pela capacidade de realizarem análises qualitativas e quantitativas em amostras ambientais, farmacêuticas, biológicas e em alimentos. Neste contexto, esta revisão tem como objetivo comentar os principais aspectos da validação em métodos cromatográficos e eletroforéticos, mostrando as diferenças e similaridades entre as diretrizes estabelecidas pelas diferentes agências reguladoras internacionais e do Brasil.

\section{CONCEITOS BÁSICOS DO PROCESSO DE VALIDAÇÃO}

Vários autores definem validação de métodos e pode-se dizer que os conceitos continuam evoluindo e estão constantemente sob consideração pelas agências reguladoras. Algumas definições podem ser transcritas:

*e-mail: icsfj@iqm.unicamp.br
- "A validação deve garantir, através de estudos experimentais, que o método atenda às exigências das aplicações analíticas, assegurando a confiabilidade dos resultados" (ANVISA)

- "Validação é o processo de definir uma exigência analítica e confirmar que o método sob investigação tem capacidade de desempenho consistente com o que a aplicação requer" (Eurachem Working Group) ${ }^{9}$.

- "Confirmação por testes e apresentação de evidências objetivas de que determinados requisitos são preenchidos para um dado uso intencional" (ISO/IEC 17025) ${ }^{13}$.

- A validação de métodos assegura a credibilidade destes durante o uso rotineiro, sendo algumas vezes mencionado como o "processo que fornece uma evidência documentada de que o método realiza aquilo para o qual é indicado para fazer" (USP) ${ }^{14}$.

- "Avaliação sistemática de um procedimento analítico para demonstrar que está sob as condições nas quais deve ser aplicado" (WHO) $)^{15}$

Vários artigos e revisões têm sido publicados a respeito de validação de métodos analíticos ${ }^{10,12,16-24}$, os quais descrevem definições, procedimentos, parâmetros e estratégias de validação.

Dentro do âmbito geral de validação de métodos é possível distinguir dois tipos ${ }^{10,21,25,26}$ :

O primeiro, chamado de validação no laboratório ("in house validation"), consiste das etapas de validação dentro de um único laboratório, seja para validar um método novo que tenha sido desenvolvido localmente ou para verificar que um método adotado de outras fontes está bem aplicado. A validação no laboratório é utilizada nas etapas preliminares do desenvolvimento de uma metodologia e na publicação de artigos para revistas científicas, em que são avaliadas todas as características de desempenho da validação da metodologia, porém sem verificar a reprodutibilidade. Pode-se considerar esta etapa como sendo preliminar à validação completa ("full validation") ${ }^{10}$.

O segundo tipo, validação completa, envolve todas as características de desempenho e um estudo interlaboratorial que é utilizado para verificar como a metodologia se comporta com uma determinada matriz em vários laboratórios, estabelecendo a reprodutibilidade da metodologia e a incerteza expandida associada à metodologia como um todo. Só assim a metodologia pode ser aceita como uma 
metodologia oficial para uma determinada aplicação. Protocolos internacionalmente aceitos têm sido estabelecidos para a validação completa, mais precisamente o Protocolo Harmonizado Internacional $^{27}$ e o procedimento ISO ("International Standard Organization") 28 . Estes protocolos requerem um número mínimo de laboratórios e materiais testes para serem incluídos no estudo interlaboratorial para validação completa do método analítico. No Brasil os estudos de comparações interlaboratoriais são coordenados pelo Instituto de Pesquisas Tecnológicas (IPT), através do Programa Brasileiro de Metrologia em Química.

\section{LEGISLAÇÃO}

Existem razões legais, técnicas e comerciais que justificam a implantação da validação de métodos analíticos de separação, apesar de não haver uma norma estabelecida de âmbito nacional ou internacional. Atualmente, para mostrar competência técnica, os laboratórios que executam as análises devem submeter-se a um credenciamento ("accreditation") de um órgão vigente de âmbito nacional ou internacional.

No Brasil, há duas agências credenciadoras para verificar a competência de laboratórios de ensaios, a ANVISA (Agência Nacional de Vigilância Sanitária) e o INMETRO (Instituto Nacional de Metrologia, Normalização e Qualidade Industrial). Estes órgãos disponibilizam guias para o procedimento de validação de métodos analíticos, respectivamente, a Resolução ANVISA RE no 899, de 29/ 05/2003 ${ }^{4}$ e o documento INMETRO DOQ-CGCRE-008, de março/ $2003^{11}$. Suas similaridades e diferenças podem ser melhor visualizadas na Tabela 1.

É importante esclarecer que resoluções são documentos com poder de lei, que devem ser obedecidas e guias são documentos que sugerem uma linha a ser seguida e são, portanto, abertos para interpretação. Os guias são recomendações e são intencionalmente vagos para deixar aos analistas a flexibilidade de adaptá-los de acordo com o método a ser usado ${ }^{29}$.

Os parâmetros para validação de métodos têm sido definidos em diferentes grupos de trabalho de organizações nacionais ou internacionais. Infelizmente algumas definições são diferentes entre as diversas organizações. Uma tentativa para harmonizar estas diferenças foi feita para aplicações farmacêuticas, através da ICH ("International Conference on Harmonization") ${ }^{2,3}$, na qual representantes das indústrias e agências reguladoras dos EUA, Europa e Japão definiram parâmetros, requerimentos e, em alguns casos, também metodologias para validação dos métodos analíticos.
A IUPAC ("International Union of Pure and Applied Chemistry") também redigiu um documento técnico que define um guia para validação de métodos analíticos que tem sido utilizado pela $\mathrm{ISO}^{10}$. A norma internacional ISO/IEC 17025, que é uma norma específica para laboratórios de ensaio e de calibração, no item 5.4.5, apresenta a "validação de métodos" como um dos requisitos técnicos importantes na qualidade assegurada dos laboratórios de ensaio, bem como a documentação do trabalho de validação ${ }^{13}$. O US-FDA ("United States Food and Drug Administration") também tem proposto guias sobre validação de métodos ${ }^{30,31}$.

Assim, órgãos como ICH, IUPAC, ISO, ANVISA, INMETRO e outros exigem o item validação de métodos analíticos como um requisito fundamental no credenciamento para qualidade assegurada e demonstração de competência técnica ${ }^{2-7,10,11}$. O que se pode observar é que não há um procedimento normatizado que estabeleça como executar a validação de métodos instrumentais de separação. Como estes organismos são responsáveis por acompanhar e credenciar a competência de laboratórios de ensaios, é importante ressaltar que as diferentes terminologias e até algumas características de desempenho do método têm, em sua maior parte, o mesmo significado, porém descrito de uma maneira distinta, para aplicações diferentes.

\section{PROCESSO DE VALIDAÇÃO}

É essencial que os estudos de validação sejam representativos e conduzidos de modo que a variação da faixa de concentração e os tipos de amostras sejam adequados. Um método para um composto majoritário requer um critério de aceitação e uma abordagem diferente de um método desenvolvido para análise de traços. A freqüência com que o método será utilizado (muitas vezes em um dia, uma vez em um dia para um estudo rápido, uma vez em um mês, etc.) também influencia o tipo de estudo de validação que é necessário. Os parâmetros analíticos devem ser baseados na intenção do uso do método. Por exemplo, se um método será usado para análise qualitativa em nível de traços, não há necessidade de testar e validar a linearidade do método sobre toda a faixa linear dinâmica do equipamento. O objetivo do método pode incluir também os diferentes tipos de equipamentos e os lugares em que o método será utilizado, ou seja, se o método é desenvolvido para ser utilizado em instrumento e laboratório específicos, não há necessidade de usar instrumentos de outras marcas ou incluir outros laboratórios nos experimentos de validação. Desta forma, os experimentos podem ser limitados para o que realmente é necessário.

Tabela 1. Parâmetros de validação do INMETRO $^{11}$ e ANVISA ${ }^{4}$

INMETRO

Especificidade/Seletividade

Faixa de trabalho e Faixa linear de trabalho

Linearidade

Limite de Detecção (LD)

Limite de Quantificação (LQ)

Sensibilidade (inclinação da curva)

Exatidão e tendência (bias)

Precisão

Repetitividade

Precisão Intermediária

Reprodutibilidade

Robustez

Incerteza de medição

ANVISA

Especificidade/Seletividade

Intervalos da curva de calibração

Linearidade

Curva de Calibração

Limite de Detecção (LD)

Limite de Quantificação (LQ)

Exatidão

Precisão

Repetibilidade (precisão intra-corrida)

Precisão intermediária (precisão inter-corrida)

Reprodutibilidade (precisão inter-laboratorial)

Robustez 


\section{PARÂMETROS ANALÍTICOS PARA VALIDAÇÃO DE MÉTODOS}

Os parâmetros analíticos normalmente encontrados para validação de métodos de separação são: seletividade; linearidade e faixa de aplicação; precisão; exatidão; limite de detecção; limite de quantificação e robustez.

Estes termos são conhecidos como parâmetros de desempenho analítico $^{22}$, características de desempenho ${ }^{10,11}$ e, algumas vezes, como figuras analíticas de mérito ${ }^{22}$.

\section{Seletividade}

A seletividade de um método instrumental de separação é a capacidade de avaliar, de forma inequívoca, as substâncias em exame na presença de componentes que podem interferir com a sua determinação em uma amostra complexa. A seletividade avalia o grau de interferência de espécies como outro ingrediente ativo, excipientes, impurezas e produtos de degradação, bem como outros compostos de propriedades similares que possam estar, porventura, presentes. A seletividade garante que o pico de resposta seja exclusivamente do composto de interesse $^{14,32}$. Se a seletividade não for assegurada, a linearidade, a exatidão e a precisão estarão seriamente comprometidas.

$\mathrm{O}$ mesmo significado tem sido freqüientemente utilizado para o termo especificidade ${ }^{3,12,14,20,21}$. Esta situação gera confusão desnecessária e isto pode ser evitado utilizando somente o termo seletividade, como sugerido pela IUPAC ${ }^{32}$. Um método instrumental de separação que produz resposta para uma única substância de interesse, normalmente um dado elemento, pode ser chamado de específico e um método que produz resposta para vários compostos químicos, com uma característica em comum, pode ser chamado de seletivo ${ }^{16,20}$. Desde que há poucos métodos cromatográficos que respondem a apenas uma substância, o termo seletividade é mais apropriado, como sugerido pela IUPAC.

A seletividade é o primeiro passo no desenvolvimento e validação de um método instrumental de separação e deve ser reavaliada continuamente durante a validação e subseqüiente uso do método. Algumas amostras podem sofrer degradação, gerando compostos que não foram observados inicialmente, que podem coeluir com a substância de interesse.

A seletividade pode ser obtida de várias maneiras. A primeira forma de se avaliar a seletividade é comparando a matriz isenta da substância de interesse e a matriz adicionada com esta substância (padrão), sendo que, nesse caso, nenhum interferente deve eluir no tempo de retenção da substância de interesse, que deve estar bem separada dos demais compostos presentes na amostra ${ }^{1,3,12,22}$. Uma segunda maneira é através da avaliação com detectores modernos (arranjo de diodos, espectrômetro de massas), que comparam o espectro do pico obtido na separação com o de um padrão e utiliza-se isto como uma indicação da presença do composto puro ${ }^{16,18,32}$. Estas duas maneiras são as mais utilizadas. O método de adição padrão também pode ser aplicado para os estudos de seletividade ${ }^{14,18}$, porém este método é utilizado quando não é possível obter a matriz isenta da substância de interesse. Neste caso é feita uma curva analítica com adição da substância de interesse na amostra e comparada com uma curva analítica sem a presença da matriz. Comparam-se então as duas curvas analíticas e caso elas sejam paralelas, pode-se dizer que não há interferência da matriz na determinação da substância de interesse, portanto o método é seletivo. Outro procedimento para avaliar a seletividade é através da coleta do composto de interesse e realização de nova análise por outra técnica cromatográfica, ou com métodos e técnicas que são específicos para a estrutura da substância de interesse como, por exemplo, espectrometria de massas, resso- nância magnética nuclear, espectroscopia no infravermelho ou bioensaios específicos ${ }^{18}$.

\section{Linearidade e faixa de aplicação}

A linearidade corresponde à capacidade do método em fornecer resultados diretamente proporcionais à concentração da substância em exame, dentro de uma determinada faixa de aplicação ${ }^{3,14,22}$.

A correlação entre o sinal medido (área ou altura do pico) e a massa ou concentração da espécie a ser quantificada muito raramente é conhecida a priori. Na maior parte dos casos, a relação matemática entre o sinal e a concentração ou massa da espécie de interesse deve ser determinada empiricamente, a partir de sinais medidos para massas ou concentrações conhecidas dessa espécie ${ }^{33}$. Essa relação matemática, muitas vezes, pode ser expressa como uma equação de reta chamada de curva analítica ${ }^{34}$. Um exemplo de curva analítica pode ser visto na Figura 1a. Embora somente dois pontos definam uma reta, na prática as linhas devem ser definidas por no mínimo cinco pontos que não incluam o ponto zero na curva, devido aos possíveis erros associados ${ }^{10}$.

Matematicamente, a estimativa dos coeficientes de uma curva analítica a partir de um conjunto de medições experimentais pode ser efetuada usando o método matemático conhecido como regressão linear ${ }^{35}$. Além dos coeficientes de regressão $\mathbf{a}$ e $\mathbf{b}$, também é possível calcular, a partir dos pontos experimentais, o coeficiente de correlação $\mathbf{r}^{36}$. Este parâmetro permite uma estimativa da qualidade da curva obtida, pois quanto mais próximo de 1,0 , menor a dispersão do conjunto de pontos experimentais e menor a incerteza dos coeficientes de regressão estimados. Para verificar se a equação de regressão é estatisticamente significativa podem ser efetuados os testes de ajuste do modelo linear, validade da regressão, sua eficiência e sua eficiência máxima ${ }^{36,37}$. Um coeficiente de correlação maior que 0,999 é considerado como evidência de um ajuste ideal dos dados para a linha de regressão $0^{12,18,38}$. A ANVISA ${ }^{4}$ recomenda um coeficiente de correlação igual a 0,99 e o INMETRO ${ }^{11}$ um valor acima de 0,90 .

Em qualquer técnica instrumental, a relação linear simples, descrita pela equação $\mathbf{y}=\mathbf{a x}+\mathbf{b}$, só é válida em um determinado intervalo de massa ou concentração da espécie medida. Este intervalo de massas ou concentrações, no qual se pode construir uma curva analítica linear, é a faixa linear dinâmica ${ }^{33}$. Ainda que as causas para a perda de linearidade sejam características de cada técnica, este é um fenômeno que pode ocorrer com qualquer conjunto de dados. Assim, o cálculo dos coeficientes de regressão de uma curva analítica deve ser acompanhado de uma cuidadosa inspeção, para verificar se todos os pontos a serem usados estão dentro da faixa linear dinâmica correspondente. Augusto et al..$^{33}$ descreveram uma forma de calcular se os pontos de uma curva analítica estão inseridos na faixa linear, baseando-se em relações geométricas do gráfico de regressão. Uma terceira abordagem é dividir os dados do sinal pelas suas respectivas concentrações, fornecendo as respostas relativas ${ }^{16}$. Um gráfico é construído com as respostas relativas no eixo y e as concentrações correspondentes em escala logarítmica no eixo x. A linha obtida deve ser horizontal sobre toda a faixa linear. São desenhadas outras linhas horizontais paralelas no gráfico, para 95 e $105 \%$ da linha da faixa linear. Conclui-se que o método é linear até o ponto onde a resposta relativa intercepta a linha de 95 ou 105\%. A Figura 1 mostra uma comparação da determinação do intervalo linear dinâmico através da curva analítica clássica (Figura 1a) e da sua representação logarítmica (Figura 1b). A construção da curva com a concentração em escala logarítmica permite melhor visualização da faixa linear.

A faixa de aplicação corresponde ao intervalo entre o valor superior e inferior da substância em exame, que atenda aos requisitos 


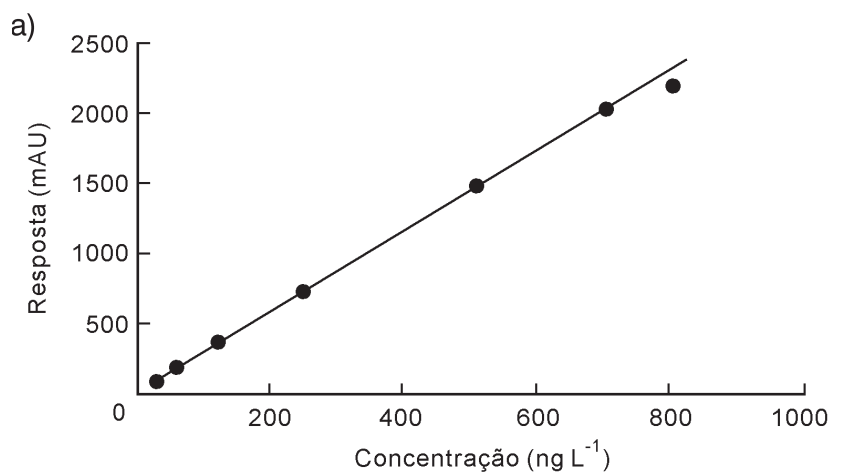

b)

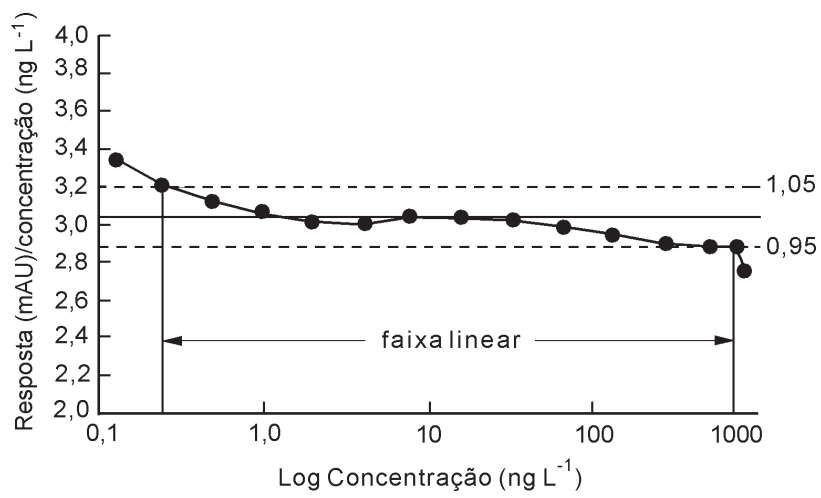

Figura 1. Determinação gráfica das curvas de linearidade através da: (a) curva analítica clássica; (b) gráfico da razão sinal/concentração vs. concentração em escala logarítmica

de precisão e exatidão ${ }^{22}$. A faixa de aplicação é normalmente expressa nas mesmas unidades dos resultados obtidos pelo método, e depende do uso em questão. Várias recomendações são encontradas na literatura. Por exemplo, a ANVISA especifica um intervalo compreendido entre $80-120 \%$ da concentração teórica para fármacos e medicamentos e de até $120 \%$ do limite máximo especificado para determinação de impurezas ${ }^{4}$. Para resíduos, o GARP (Associação Grupo de Analistas de Resíduos de Pesticidas) ${ }^{39}$ recomenda uma faixa de concentração com valores variando entre a metade e o quíntuplo da concentração do limite de quantificação. A IUPAC especifica que os pontos da curva analítica devem ser igualmente espaçados sobre a faixa de concentração de interesse e que esta faixa compreenda 0 $150 \%$ ou $50-150 \%$ do valor esperado, dependendo de qual destas duas opções for mais adequada ${ }^{10}$. Para produtos formulados, a ICH, entre outros, recomenda uma variação de $\pm 20 \%$ do valor declarado ou esperado ${ }^{3,7,12}$.

As diretrizes da $\mathrm{ICH}^{3}$ e da ANVISA ${ }^{4}$ especificam um mínimo de cinco níveis de concentração, juntamente com certos mínimos de variação especificados. O GARP também sugere cinco concentrações que devem ser injetadas em ordem crescente de concentração, no mínimo três vezes cada, com estimativa do desvio padrão relativo (RSD) entre as injeções inferior a 5\%. A IUPAC recomenda seis ou mais níveis de concentração ${ }^{10}$.

A quantificação do composto de interesse em validação pode ser obtida através dos seguintes métodos: padronização externa; padronização interna; superposição de matriz; adição padrão.

\section{Padronização externa}

O método de padronização externa compara a área da substância a ser quantificada na amostra com as áreas obtidas com soluções de concentrações conhecidas preparadas a partir de um padrão. Preparam-se soluções da substância a ser quantificada em diversas con- centrações; obtém-se o cromatograma correspondente a cada uma delas e, em um gráfico, relacionam-se as áreas obtidas com as concentrações. Utilizando este gráfico ou a equação da curva resultante, pode-se calcular a concentração desta substância na amostra a partir da área da substância obtida no cromatograma resultante de uma injeção separada. Este método é sensível a erros de preparo das amostras e dos padrões e de injeção das soluções padrão e das amos$\operatorname{tras}^{40,41}$ e por isso deve ser feito a cada análise.

\section{Padronização interna}

O método de padronização interna consiste na preparação das soluções padrão de concentrações conhecidas da substância de interesse, às quais se adiciona a mesma quantidade conhecida de um composto chamado padrão interno. Após análise dessas soluções, constrói-se um gráfico, relacionando a razão de áreas (área da substância/área do padrão interno que tem concentração constante) com a concentração (variada) da substância. A amostra também é analisada após a adição da mesma quantidade conhecida do padrão interno. Através da razão de áreas obtidas no cromatograma tem-se a concentração da substância na amostra. Idealmente, a substância usada como padrão interno deve ser similar à substância a ser quantificada, ter tempo de retenção próximo a esta substância, não reagir com a substância ou outro componente da matriz, não fazer parte da amostra e, quando cromatografada, ficar separada de todas as demais substâncias presentes na amostra ${ }^{40,41}$. Este último requisito não é necessário quando a detecção é feita por espectrometria de massas, na qual cada composto produz um espectro característico. O método de padronização interna é extremamente útil, especialmente pelo fato de que independe de pequenas mudanças em variáveis experimentais, como temperatura da coluna e tamanho da amostra. Este método é bastante útil em cromatografia gasosa, na qual se usa seringa para injeção de amostra ${ }^{42}$ e por isso deve ser feito a cada análise.

\section{Superposição de matriz.}

O método de superposição de matriz ("matrix-matched") consiste na adição do padrão da substância em diversas concentrações em uma matriz similar à da amostra, isenta da substância, e construção do gráfico de calibração relacionando as áreas obtidas com as concentrações dos padrões. O método de superposição de matriz pode ser utilizado para calibração, tanto com a padronização interna como com a padronização externa. Este método é usado para compensar o efeito da matriz ou de possíveis interferentes e é de suma importância em determinações quando a matriz pode interferir na pré-concentração, extração, separação ou detecção da substância de interesse. Sua principal vantagem sobre o método de padronização externa é que fornece uma melhor correspondência com a composição da amostra. Por exemplo, se algumas substâncias são determinadas em soro humano e uma solução padrão aquosa for usada na calibração, resultados errôneos podem ser obtidos por causa do efeito da matriz; para tais medições, uma matriz de soro humano seria melhor para realizar a calibração do que a solução aquosa ${ }^{41}$. O método de superposição de matriz tem o inconveniente de não proporcionar a magnitude do efeito de co-extratos, além de aumentar o custo e o tempo das análises ${ }^{43}$. Alguns autores acreditam que o efeito dos coextratos sobre a resposta da substância de interesse deveria ser avaliado pela comparação do método de superposição de matriz com a padronização externa (padrões preparados nos solventes) ${ }^{25}$. Apesar de se obter uma calibração confiável com o método de superposição da matriz, ele é somente uma forma para compensar efeitos da matriz, mas não elimina situações analíticas típicas: a intensidade de um efeito e a concentração de interferentes na matriz podem diferir de uma matriz ou amostra para outra ${ }^{44}$. Assim, em amostras nas quais pode ocorrer o efeito da matriz e não se tem disponível uma matriz 
isenta da substância de interesse para utilizar o método de superposição de matriz, deve-se utilizar o método de adição padrão ${ }^{44}$.

\section{Adição padrão}

O método de adição padrão consiste na adição de quantidades conhecidas da substância de interesse que está sendo analisada a quantidades conhecidas da amostra, antes do seu preparo. Estas amostras com o padrão incorporado são utilizadas para a obtenção dos cromatogramas. Constrói-se uma curva analítica relacionando as quantidades da substância adicionada à amostra com as respectivas áreas obtidas. O ponto onde a reta corta o eixo das ordenadas corresponde à área do pico da substância que está sendo determinada, sem qualquer adição do padrão. A extrapolação da reta define, no eixo das abcissas, a concentração da substância na amostra analisada ${ }^{45}$. O método de adição padrão é trabalhoso, mas é especialmente importante quando a amostra é muito complexa, quando as interações com a matriz são significativas e quando houver dificuldade de encontrar um padrão interno adequado ou uma matriz isenta da substância de interesse $\mathrm{e}^{24}$.

A Figura 2 mostra a interrelação entre o método de adição padrão, superposição de matriz e padronização externa. O comportamento paralelo entre as linhas de regressão mostra que o método tem seletividade. De forma geral, pode-se dizer que o método de padronização externa é realizado quando nenhum erro sistemático proveniente da matriz é suspeito, enquanto o método de superposição de matriz compensa o efeito da matriz e o método de adição padrão corrige o efeito da matriz e as mudanças da resposta do instrumento.

Os métodos de quantificação não têm regras ou guias, exceto que o método final selecionado deve fornecer a melhor exatidão possível e um alto nível de precisão. O método escolhido para quantificação deve atingir estes objetivos em um menor tempo possível, com um mínimo de envolvimento do operador, além de utilizar pouca quantidade de amostra. O método de quantificação ideal dependerá da amostra específica, do número de amostras, da complexidade da matriz, da possibilidade de automação e da disponibilidade de padrões.

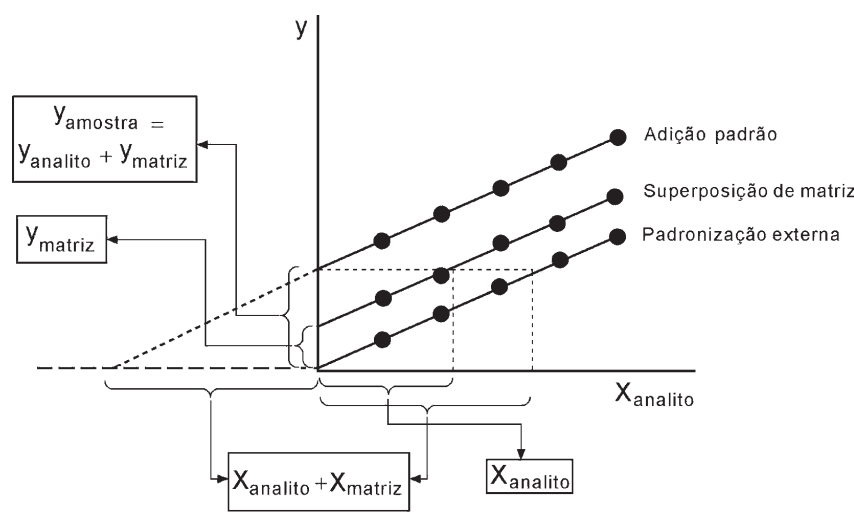

Figura 2. Interrelação entre os diferentes métodos de construção da curva analítica

Quanto aos aspectos práticos, o preparo das soluções padrão pode ser realizado de diversas maneiras. Em uma delas, prepara-se uma solução mais concentrada, a partir da pesagem do padrão, denominada solução estoque, que deve ser preparada de acordo com o tempo de estabilidade da substância em solução ou pelo menos a cada seis meses. As soluções preparadas por diluição são chamadas soluções de trabalho e recomenda-se que sejam preparadas pelo menos a cada duas ou três semanas ${ }^{39}$. Duas abordagens ocorrem no preparo das soluções por diluição. Na primeira, as soluções de trabalho po- dem partir de uma única solução estoque, através de diluições sucessivas das soluções de trabalho anteriormente preparadas ou através do preparo de todas as soluções diluídas, partindo sempre da solução estoque. Este último modo é o mais recomendado, porém, dependendo da faixa de concentração em questão, diluições diretamente da solução estoque podem envolver medições de volumes tão pequenas que o erro se torna grande. A Figura 3a mostra um esquema da sequiência utilizando este modo de preparo. Uma outra abordagem para o preparo das soluções de trabalho é mostrada na Figura 3b. Neste caso, são preparadas duas soluções estoque de concentrações diferentes e as soluções de trabalho são preparadas a partir destas duas soluções, seja por diluições sucessivas ou partindo das soluções estoque. Este modo de preparo das soluções de trabalho é mais adequado, pois permite avaliar através da curva analítica o erro embutido na solução estoque, decorrente da pesagem dos padrões.

Uma outra maneira de se preparar as soluções seria através da pesagem separada de cada concentração do padrão da substância da curva analítica, ao invés da utilização de diluições sucessivas de uma solução mais concentrada ${ }^{40}$. Esta é a maneira ideal de se preparar as soluções, pois a preparação individual mostra o erro verdadeiro na

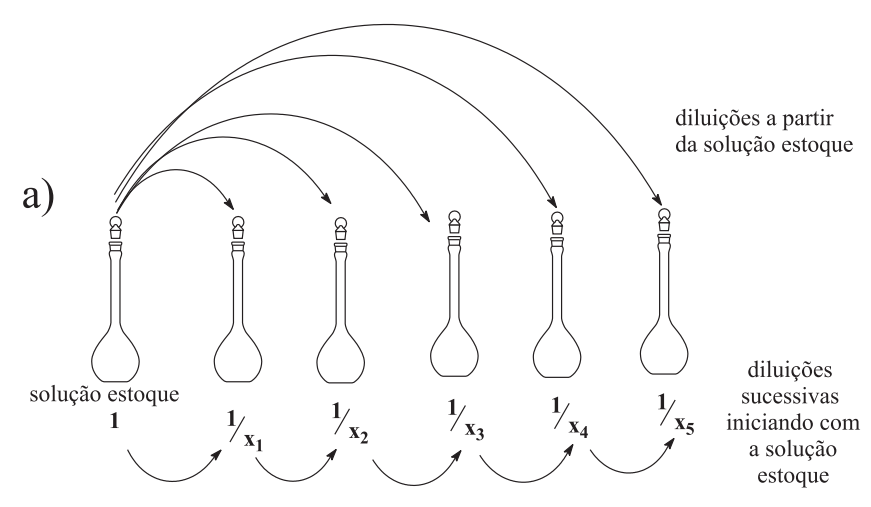

b)
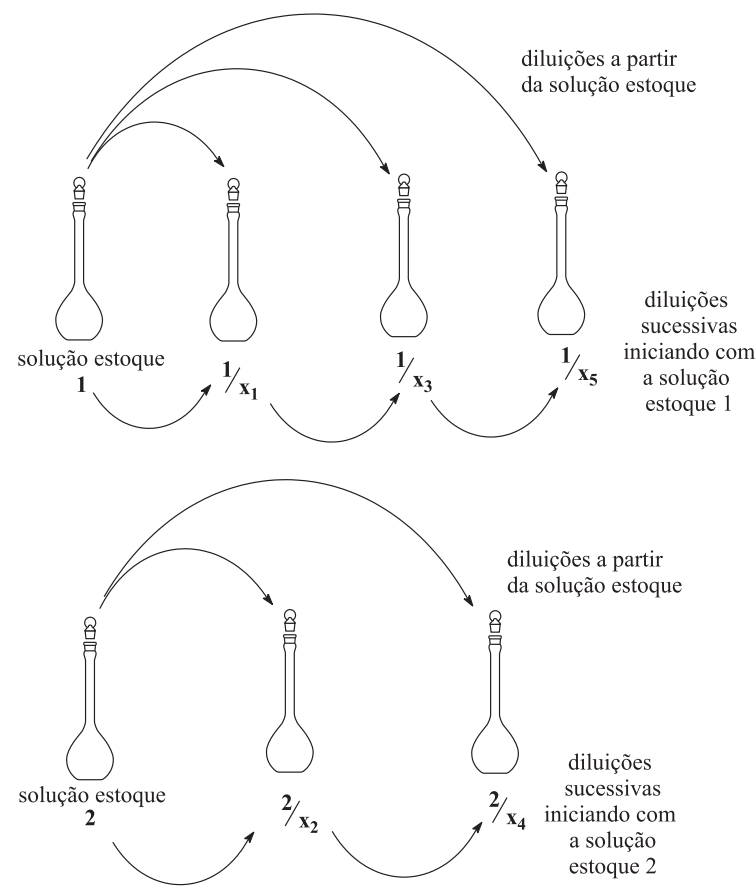

Figura 3. Modos de diluição da solução estoque: a) diluição a partir de uma solução estoque e b) diluição a partir de duas soluções estoques ( 1 e 2) preparadas com concentrações diferentes 
preparação de todas as soluções padrão e não, erros de diluição. Entretanto, este método torna-se inviável quando se trabalha com análise de traços, já que a quantidade de padrão a ser pesada é tão pequena que a sensibilidade da balança semi-microanalítica não permite tais pesagens, devido aos erros associados.

\section{Precisão}

Representa a dispersão de resultados entre ensaios independentes, repetidos de uma mesma amostra, amostras semelhantes ou padrões, sob condições definidas ${ }^{3,11}$. A precisão é avaliada pelo desvio padrão absoluto $(\sigma)$, que utiliza um número significativo de medições, normalmente maior que 20. Na prática, em validação de métodos, o número de determinações é geralmente pequeno e o que se calcula é a estimativa do desvio padrão absoluto (s).

$\mathrm{s}=\sqrt{\frac{\sum\left(\mathrm{x}_{\mathrm{i}}-\overline{\mathrm{x}}\right)^{2}}{\mathrm{n}-1}}$

$\bar{x}$ é a média aritmética de um pequeno número de medições (média das determinações), sendo uma estimativa de $\mu$, a média verdadeira (média da população); $x_{\mathrm{i}}$ é o valor individual de uma medição e n é o número de medições.

A precisão também pode ser expressa através do intervalo de confiança da média, que é uma faixa de valores no qual existe uma determinada probabilidade de se encontrar um certo valor de uma variável, calculada pela equação:

Intervalo de confiança da média $=\overline{\mathrm{x}} \pm \mathrm{t}_{\mathrm{n}-1} \frac{\mathrm{s}}{\sqrt{\mathrm{n}}}$

em que: $\mathrm{t}_{\mathrm{n}-1}=$ valor crítico da distribuição de Student com n-1 graus de liberdade. $\mathrm{O}$ valor té tabelado e apresenta valores para diferentes níveis de confiança.

Outra expressão da precisão é através da estimativa do desvio padrão relativo (RSD), também conhecido como coeficiente de variação (CV).

$\operatorname{RSD}(\%)$ ou CV $(\%)=\frac{\mathrm{s}}{\overline{\mathrm{x}}} \times 100$

Normalmente, métodos que quantificam compostos em macro quantidades requerem um RSD de 1 a $2 \%$. Em métodos de análise de traços ou impurezas, são aceitos RSD de até $20 \%$, dependendo da complexidade da amostra ${ }^{16}$. Uma maneira simples de melhorar a precisão é aumentar o número de replicatas.

A precisão em validação de métodos é considerada em três níveis diferentes: repetitividade; precisão intermediária; reprodutibilidade.

\section{Repetitividade}

A repetitividade ("repeatability") representa a concordância entre os resultados de medições sucessivas de um mesmo método, efetuadas sob as mesmas condições de medição, chamadas condições de repetitividade: mesmo procedimento; mesmo analista; mesmo instrumento usado sob as mesmas condições; mesmo local; repetições em um curto intervalo de tempo. O termo repetitividade é adotado pelo Vocabulário Internacional de Metrologia ${ }^{46}$, sendo utilizado pelo INMETRO. Por outro lado, a ANVISA utiliza o mesmo conceito para o termo repetibilidade 4 .

A repetitividade envolve várias medições da mesma amostra, em diferentes preparações e é, algumas vezes, denominada precisão intraensaio $^{12,38}$ ou intra-corrida ${ }^{4}$ e pode ser expressa através da estimativa do desvio padrão relativo (RSD).

Não se deve confundir repetitividade com precisão instrumental, a qual é medida pelas injeções repetitivas, seqüenciais da mesma amostra (tipicamente 10 ou mais vezes), seguida pela média dos valores da área do pico ou altura do pico e determinação da estimativa do desvio padrão relativo de todas as injeções.

Para a repetitividade, o INMETRO ${ }^{11}$ recomenda sete ou mais repetições para o cálculo da estimativa do desvio padrão. ${\mathrm{A} \mathrm{ICH}^{3} \mathrm{e}}$ ANVISA $^{4}$ sugerem que a repetitividade seja verificada a partir de um mínimo de nove determinações cobrindo o limite especificado do procedimento (ex.: três níveis, três repetições cada um), ou a partir de um mínimo de seis determinações a uma concentração similar ao valor esperado.

\section{Precisão intermediária}

Indica o efeito das variações dentro do laboratório devido a eventos como diferentes dias ou diferentes analistas ou diferentes equipamentos ou uma combinação destes fatores ${ }^{3}$.

A precisão intermediária é reconhecida como a mais representativa da variabilidade dos resultados em um único laboratório e, como tal, mais aconselhável de ser adotada. O objetivo da validação da precisão intermediária é verificar que no mesmo laboratório o método fornecerá os mesmos resultados.

O número de ensaios necessários para se avaliar a precisão intermediária segue a mesma recomendação da $\mathrm{ICH}^{3}$ e $\mathrm{ANVISA}^{4}$ para o cálculo de repetitividade descrita acima. A precisão intermediária pode ser expressa através da estimativa do desvio padrão relativo (RSD).

\section{Reprodutibilidade}

É o grau de concordância entre os resultados das medições de uma mesma amostra, efetuadas sob condições variadas (mudança de operador, local, equipamentos, etc. $)^{46}$. A reprodutibilidade refere-se aos resultados dos estudos de colaboração entre laboratórios e deve ser considerada em situações como a padronização de procedimentos analíticos a serem incluídos, por exemplo, em farmacopéias, procedimentos do CODEX, etc. É muito comum encontrar desacordo entre métodos analíticos. Isto aparece quando vários laboratórios analisam uma amostra em comum, em estudos colaborativos. Freqüentemente, altas variações são observadas entre os resultados. Assim, os dados provenientes de apenas um laboratório não são suficientes para avaliar a reprodutibilidade do método. Estudos colaborativos não são somente indispensáveis para avaliação da reprodutibilidade, eles também podem ser de grande ajuda para testar a exatidão do método ${ }^{47}$.

A IUPAC não aconselha tirar conclusões com menos de cinco laboratórios e recomenda oito laboratórios em seu guia atual ${ }^{10}$. Além disso, mais crítico que o número de laboratórios envolvidos é que estes possuam competência e habilidades similares àqueles que usarão o método em rotina.

A documentação que apóia os estudos de precisão em nível de reprodutibilidade deve incluir estimativa do desvio padrão absoluto, estimativa do desvio padrão relativo e intervalo de confiança. Horwitz et al..$^{48}$ estabeleceram uma relação matemática para expressar a dependência entre valores do RSD e concentração da substância, pelo exame de resultados cumulativos de estudos colaborativos envolvendo grande faixa de compostos de interesse, matrizes e técnicas analíticas. Os valores obtidos por esta relação matemática são introduzidos em um gráfico e originam a denominada Trombeta de Horwitz ${ }^{48}$.

\section{Exatidão}

Representa o grau de concordância entre os resultados individuais encontrados em um determinado ensaio e um valor de referência aceito como verdadeiro ${ }^{3,11}$. É importante observar que um valor exato ou verdadeiro é o valor obtido por uma medição perfeita e este valor é indeterminado por natureza ${ }^{49}$. 
A exatidão é sempre considerada dentro de certos limites, a um dado nível de confiança (ou seja, aparece sempre associada a valores de precisão). Estes limites podem ser estreitos em níveis de concentração elevados e mais amplos em níveis de traços.

O número de ensaios varia segundo a legislação ou diretriz adotada e também com as características da pesquisa. $\mathrm{A} \mathrm{ICH}^{3}$ estabelece que um mínimo de nove determinações envolvendo um mínimo de três diferentes níveis de concentração deve ser obedecido. Por exemplo, ensaios em triplicata para três níveis de concentração. Esta recomendação é também adotada pela ANVISA ${ }^{4}$.

Os processos mais utilizados para avaliar a exatidão de um método são: materiais de referência; comparação de métodos; ensaios de recuperação; adição padrão.

\section{Materiais de referência certificados (CRM)}

Os CRM são materiais de referência acompanhados de um certificado que possui o valor de concentração de uma dada substância, ou outra grandeza para cada parâmetro e uma incerteza associada. Os materiais de referência certificados são fornecidos por organismos reconhecidos e confiáveis, como NIST ("National Institute of Standards and Technology" - USA), LGC ("Laboratory of the Government Chemist" - UK), USP, FAPAS ("Food Analysis Performance Assessment Scheme" - UK), etc.

Os valores obtidos pelo laboratório (a média e a estimativa do desvio padrão de uma série de replicatas) da mesma amostra padrão devem ser comparados com os valores certificados do material de referência, para verificar a exatidão do método.

\section{Comparação de métodos}

Consiste na comparação entre resultados obtidos empregandose o método em desenvolvimento e os resultados conseguidos através de um método de referência, avaliando o grau de proximidade entre os resultados obtidos pelos dois métodos, ou seja, o grau de exatidão do método testado em relação ao de referência. Esta abordagem assume que a incerteza do método de referência é conhecida.

As análises são efetuadas em replicata, utilizando os dois métodos em separado (o método em desenvolvimento e o método de referência), sobre as mesmas amostras, em uma faixa de concentrações em que se pretende validar o método.

\section{Ensaios de recuperação}

A recuperação (ou fator de recuperação), $\mathrm{R}$, é definida como a proporção da quantidade da substância de interesse, presente ou adicionada na porção analítica do material teste, que é extraída e passível de ser quantificada ${ }^{50}$

A informação de recuperação pode ser estimada de CRM (em que a quantidade de substância é previamente conhecida), quando disponíveis, ou de um composto substituto ("surrogate”). O substituto é definido como um composto ou elemento puro adicionado ao material teste, no qual o comportamento químico e físico é representativo da substância de interesse na forma nativa ${ }^{41}$. Diz-se que o composto é um substituto porque este é transferido para a amostra e pode não estar efetivamente no mesmo equilíbrio que se encontra a substância na forma nativa, então determina-se a recuperação do substituto, fazendo uma "correção de recuperação" para a substância de interesse $\mathrm{e}^{50}$. Os compostos substitutos, adicionados nas amostras, podem ser de vários tipos:

- padrão da substância adicionado à matriz isenta da substância ou à amostra (fortificação, incorporação, dopagem, enriquecimento, termos provenientes do inglês "spiking"); o US-FDA reconhece duas categorias de padrões de referência: compendiais e não compendiais. Os padrões de referência compendiais são obtidos de fontes como a USP e não necessitam de caracteriza- ção posterior. Os padrões de referência não compendiais são substâncias com elevado teor de pureza, que podem ser obtidas através de um esforço razoável e devem ser cuidadosamente caracterizados para garantir sua identidade, potência e pureza. É recomendável que fatores de correção de pureza sejam incluídos em qualquer cálculo existente no método.

- uma versão da substância modificada isotopicamente e

- composto quimicamente diferente da substância de interesse, mas representativo de seu comportamento. Algumas vezes este composto é denominado padrão interno ${ }^{23,41}$.

A limitação do procedimento de recuperação é a de que a substância adicionada não está, necessariamente, na mesma forma que a presente na amostra. Isso pode implicar, por exemplo, na presença de substâncias adicionadas em uma forma que proporcione melhor detecção, ocasionando avaliações excessivamente otimistas da recuperação. Pelo fato de outros componentes da matriz poderem interferir na separação, detecção ou na quantificação da substância, efeitos dos componentes da matriz devem ser investigados.

É importante considerar como a eficiência do método varia em função da concentração da substância. Na maioria dos casos, a dispersão dos resultados aumenta com a diminuição da concentração e a recuperação pode diferir substancialmente a altas e baixas concentrações. Por esse motivo, a recuperação deve ser avaliada na faixa de concentração esperada para o composto de interesse. Isto pode ser feito adicionando a substância em pelo menos três diferentes concentrações, por exemplo, próximo ao limite de quantificação, próximo à concentração máxima permitida pelo método em teste e em uma concentração próxima à média da faixa de uso do método. Para análises em nível de resíduos, o GARP recomenda que se trabalhe nos níveis de adição de 1,2 e 10 vezes o valor de limite de quantificação ${ }^{39}$. Para componentes em maiores concentrações, os níveis de adição podem ser 50, 75, 100, 125 e $150 \%$ do nível esperado para a substância ${ }^{24}$.

As medições de recuperação são as mais comuns devido à dificuldade em se obterem CRM (que, para certas aplicações, nem existem) e são expressas em termos de porcentagem da quantidade medida da substância em relação à quantidade adicionada na matriz (branco ou placebo), em um determinado número de ensaios ${ }^{51}$.

Os intervalos aceitáveis de recuperação para análise de resíduos geralmente estão entre 70 e $120 \%$, com precisão de até $\pm 20 \%{ }^{39}$. Porém, dependendo da complexidade analítica e da amostra, este valor pode ser de 50 a $120 \%$, com precisão de até $\pm 15 \%$.

\section{Adição padrão}

Este método é usado quando for difícil ou impossível preparar um branco da matriz sem a substância de interesse. Um exemplo seria a análise de $\beta$-caroteno em amostras de mamão, nas quais o $\beta$ caroteno sempre estará presente.

No método de adição padrão, quantidades conhecidas da substância são adicionadas em diferentes níveis numa matriz da amostra, antes do procedimento de preparo da amostra, que já contenha quantidades (desconhecidas) da substância ${ }^{52}$. A concentração da substância de interesse na amostra original pode ser determinada gráfica e matematicamente, como já mostrado anteriormente. Em geral, para adição padrão, uma boa abordagem é adicionar 25, 50 e 100\% da concentração esperada da substância na matriz ${ }^{24}$. A amostra sem adição do padrão e cada uma das amostras com o padrão adicionado devem ser analisadas e as quantidades medidas relacionadas com a quantidade adicionada.

\section{Limite de Detecção (LD)}

O limite de detecção (LD) representa a menor concentração da substância em exame que pode ser detectada, mas não necessaria- 
mente quantificada, utilizando um determinado procedimento experimental ${ }^{3,11}$

O LD pode ser calculado de três maneiras diferentes: método visual, método relação sinal-ruído, método baseado em parâmetros da curva analítica.

\section{Método visual}

É utilizado para determinar o limite de detecção utilizando a matriz com adição de concentrações conhecidas da substância de interesse, de tal modo que se possa distinguir entre ruído e sinal analítico pela visualização da menor concentração visível (detectável). Este procedimento também pode ser feito através do instrumento utilizando parâmetros de detecção no método de integração.

\section{Método da relação sinal-ruído}

Este método pode ser aplicado somente em procedimentos analíticos que mostram o ruído da linha de base. Para determinar a relação sinal-ruído, é feita a comparação entre a medição dos sinais de amostras em baixas concentrações conhecidas do composto de interesse na matriz ${ }^{39} \mathrm{e}$ um branco (matriz isenta do composto de interesse) destas amostras. Assim, é estabelecida uma concentração mínima na qual a substância pode ser facilmente detectada. A relação sinal-ruído pode ser de $3: 1$ ou 2:1, proporções geralmente aceitas como estimativas do limite de detecção.

Método baseado em parâmetros da curva analítica

O limite de detecção (LD) pode ser expresso como:

$\mathrm{LD}=3,3 \times \frac{s}{\mathrm{~S}}$

onde s é a estimativa do desvio padrão da resposta, que pode ser a estimativa do desvio padrão do branco, da equação da linha de regressão ou do coeficiente linear da equação e S é a inclinação ("slope") ou coeficiente angular da curva analítica.

Para calcular estes dados, uma curva analítica deverá ser feita utilizando a matriz contendo o composto de interesse na faixa de concentração próxima ao limite de detecção. Softwares como Microsoft Excel ${ }^{\circledR}$ ou Microcal Origin ${ }^{\circledR}$ podem calcular os parâmetros da curva e a estimativa do desvio padrão relativo destes parâmetros. Para adquirir melhor compreensão dos cálculos envolvidos, pode-se consultar livros de estatística ${ }^{37,53,54}$.

\section{Limite de Quantificação (LQ)}

O limite de quantificação (LQ) representa a menor concentração da substância em exame que pode ser medida, utilizando um determinado procedimento experimental ${ }^{3,11}$.

Como o LD, o LQ é expresso como uma concentração, sendo que a precisão e exatidão das determinações também devem ser registradas. Esse critério é uma boa regra a ser seguida, porém não se deve esquecer que a determinação do LQ representa um compromisso entre a concentração, a precisão e a exatidão exigidas. Isto significa que, quando decresce o nível de concentração do LQ, a medição torna-se menos precisa. Se houver necessidade de maior precisão, uma concentração maior deve ser registrada para o LQ. O método analítico e seu respectivo uso ditam esse compromisso.

Os mesmos critérios de LD podem ser adotados para o LQ, utilizando a relação 10:1, ou seja, o LQ pode ser calculado utilizando o método visual, a relação sinal-ruído ou a relação entre a estimativa do desvio padrão da resposta (s) (que pode ser a estimativa do desvio padrão do branco, da equação da linha de regressão ou do coeficiente linear da equação) e a inclinação da curva analítica (S), em níveis próximos ao LQ, a partir da equação:

$\mathrm{LQ}=10 \times \frac{s}{\mathrm{~S}}$

O método mais utilizado é o da relação sinal-ruído para técnicas analíticas em geral, porém em técnicas analíticas de separação, como as cromatográficas e eletroforéticas, a medição do ruído não é trivial e às vezes subjetiva (já que a curva analítica é construída com a área e não somente o sinal do detector). Além disso, tanto o LD quanto o LQ podem ser afetados pelas condições cromatográficas. Picos maiores aumentam a relação sinal-ruído, resultando em LD e LQ mais baixos. Além disso, a determinação cromatográfica desses parâmetros deve considerar tanto o tipo quanto o tempo de uso da coluna.

O melhor caminho para resolver este problema do cálculo do LD e LQ é utilizar o método baseado nos parâmetros da curva analítica, que é estatisticamente mais confiável. A curva analítica deve conter a concentração correspondente ao LQ.

\section{Robustez}

De acordo com o INMETRO ${ }^{11}$, a robustez de um método ("robustness") mede a sensibilidade que este apresenta face a pequenas variações. Diz-se que um método é robusto quando ele não é afetado por uma modificação pequena e deliberada em seus parâmetros. A robustez de um método cromatográfico é avaliada, por exemplo, pela variação de parâmetros como a concentração do solvente orgânico, pH e força iônica da fase móvel em HPLC, programação da temperatura, natureza do gás de arraste em GC, bem como o tempo de extração, agitação, etc. As mudanças introduzidas refletem as alterações que podem ocorrer quando um método é transferido para outros laboratórios, analistas ou equipamentos ${ }^{55}$.

Para determinar a robustez de um método, o INMETRO recomenda o teste de Youden ${ }^{11}$. Trata-se de um teste que permite não só avaliar a robustez do método, como também ordenar a influência de cada uma das variações nos resultados finais, indicando qual o tipo de influência de cada uma dessas variações. Por este teste são realizados oito ensaios com uma combinação fatorial dos efeitos e verifica-se qual o efeito ou combinação de efeitos que apresentam variações.

A IUPAC ${ }^{10}$ utiliza o mesmo conceito de robustez para a palavra "ruggedness". A USP também utiliza o termo "ruggedness", mas com uma definição diferente, que lembra reprodutibilidade: "A robustez de um método analítico é o nível de reprodutibilidade dos resultados dos testes obtidos pelas análises de algumas amostras sob uma variedade de condições normais de teste, tais como diferentes laboratórios, diferentes analistas, diferentes instrumentos, diferentes lotes de reagentes, diferentes dias, etc." ${ }^{14}$.

Em HPLC, a robustez pode ser avaliada, por exemplo, variando o conteúdo de metanol na fase móvel em $\pm 2 \%$, o pH da fase móvel em 0,1 unidades de $\mathrm{pH}$ ou a temperatura da coluna em $\pm 5^{\circ} \mathrm{C}$. Se estas mudanças estiverem dentro dos limites de exatidão,e precisão e seletividade aceitáveis, então o método possui robustez e tais variações podem ser incorporadas ao procedimento.

Em trabalhos nos quais há mudanças de fornecedores, marcas ou equipamentos ao longo do desenvolvimento e validação das metodologias, sem alteração significativa nos resultados, pode-se dizer que o método possui uma robustez intrínseca, pois manteve sua resposta em meio a mudanças de ambiente de análise.

\section{ESTABILIDADE DOS PADRÕES E DAS AMOSTRAS}

Para gerar resultados confiáveis e reprodutíveis, as amostras, os padrões e reagentes usados devem ser estáveis por um período razo- 
ável (por ex. um dia, uma semana, um mês, dependendo da necessidade $)^{12}$. Freqüientemente, em equipamentos automatizados, as corridas cromatográficas são realizadas durante a noite para melhor aproveitamento do funcionamento do laboratório. Esta prática requer maior estabilidade das soluções. A estabilidade das amostras e padrões é importante em termos de temperatura e tempo. Se uma solução não for estável em temperaturas ambientes, a diminuição da temperatura pode aumentar a estabilidade das amostras e padrões. Com relação ao tempo, estabilidade de dias ou meses é mais desejável, entretanto em alguns casos, as soluções precisam ser preparadas cada vez que forem realizadas as análises ${ }^{24}$.

Em certos tipos de amostras, faz-se necessário avaliar a estabilidade da substância para determinar o tempo de estocagem das amos$\operatorname{tras}^{38}$. Tempos longos de estocagem de amostras biológicas, por exemplo, aumentam a probabilidade de degradação dos compostos de interesse, com subseqüiente formação de metabólitos. Conhecendo a estabilidade, as análises podem ser completadas antes de ocorrer a degradação.

\section{CONFORMIDADE DO SISTEMA}

Antes de realizar experimentos de validação ou mesmo análises de amostras, deve-se avaliar se o sistema utilizado para a análise é capaz de fornecer dados de qualidade aceitável. Esta avaliação é alcançada com experimentos de conformidade do sistema ("system suitability"), que pode ser definida como um conjunto de testes para garantir que o equipamento utilizado está apto a gerar resultados de exatidão e precisão aceitáveis. A conformidade do sistema pode causar dúvidas quanto ao seu alcance e, por isso, é encontrada em duas abordagens.

A primeira considera que a resolução e a repetitividade do sistema cromatográfico sejam adequadas para a análise a ser realizada. Assim, a conformidade do sistema é verificada antes que o desenvolvimento do método e a validação tenham sido completados. Os critérios selecionados são baseados no desempenho do método determinado durante a validação. Por exemplo, se o tempo de retenção da amostra fizer parte do critério de conformidade do sistema, a sua variação (estimativa do desvio padrão) pode ser determinada durante a validação, que pode ter uma variação de $3 \%$, por exemplo (baseado nos resultados de validação) durante o uso rotineiro.

A segunda abordagem considera o sistema como um todo e inclui, além do sistema cromatográfico, a calibração e manutenção dos equipamentos e instrumentos utilizados em todo o procedimento analítico, dentro das especificações. Neste caso, a conformidade do sistema baseia-se no conceito de que o equipamento, componentes eletrônicos, operações analíticas e amostras constituem um sistema integral que pode ser avaliado como um todo. Pode-se dizer, então, que a conformidade do sistema é a verificação de um sistema para garantir a sua qualidade antes ou durante a análise de amostras desconhecidas.

Alguns autores consideram que, se o sistema estiver qualificado, a validação do método pode ser desenvolvida. Algumas vezes os critérios para avaliação da conformidade do sistema são definidos antes da validação e, quando realizados durante as análises, estes testes garantem que o desempenho do sistema está apropriado para o uso.

Os parâmetros a serem medidos e seus limites recomendados, de acordo com a US-FDA, estão na Tabela $2^{6}$. Tipicamente, no mínimo dois destes critérios são requeridos para garantir a conformidade do sistema.

\section{REVALIDAÇÃO}

Dentro de um laboratório é provável que, após um período de tempo, certos reagentes e equipamentos possam ter sofrido altera-
Tabela 2. Parâmetros de conformidade do sistema e recomendações ${ }^{6}$

\begin{tabular}{|c|c|}
\hline Parâmetro & Recomendação \\
\hline Fator de retenção $(\mathrm{k})$ & $\begin{array}{l}\text { O pico deve estar bem separado } \\
\text { de outros picos e do pico } \\
\text { correspondente ao tempo de } \\
\text { retenção de um composto não } \\
\text { retido }\left(t_{M}\right), k>2\end{array}$ \\
\hline Repetitividade (RSD) & $\mathrm{RSD}<1 \%$ para $\mathrm{n}>5$ \\
\hline Resolução (Rs) & $\begin{array}{l}\text { Rs }>2 \text { entre o pico de interesse } \\
\text { e o interferente potencial mais } \\
\text { próximo (impureza, produto de } \\
\text { degradação) }\end{array}$ \\
\hline Fator de alargamento (TF) & $\mathrm{TF} \leq 2$ \\
\hline Número de pratos da coluna $(\mathrm{N})$ & $\begin{array}{l}\text { Em geral deve ser }>2000 \text { para } \\
\text { HPLC }\end{array}$ \\
\hline
\end{tabular}

ções, seja por mudança de fornecedor, troca de componentes ou desgaste do equipamento provocado pelo uso constante. É possível que o desempenho do método e, então, a validade dos resultados gerados pelo método sejam afetados por estas mudanças. A revalidação, que pode ser necessária em tal situação, é a reavaliação de um método analítico validado em resposta a uma mudança em algum aspecto do método $^{18,25}$. É impraticável e provavelmente desnecessário revalidar um método que tenha sofrido "pequenas mudanças". Propõe-se que estas pequenas variações sejam avaliadas durante a validação, no parâmetro de robustez, e que a revalidação de método seja limitada às situações como mudanças de maiores extensões. Para métodos de separação, alterações significativas poderiam ser devido a mudanças no produto para o qual o método foi validado, no instrumento, no reagente (tipo ou fabricante) ou no procedimento.

A revalidação também deve ser considerada quando a proposta e/ou o nível de qualidade desejado do método são alterados, o procedimento é modificado, ou mesmo quando um método é usado novamente após um certo período de tempo.

Com respeito a quais parâmetros devem ser inclusos na revalidação, pode-se dizer que quanto maiores as alterações no método, maior deve ser a abrangência da revalidação.

\section{CONCLUSÃO}

Os conceitos de validação de métodos continuam a evoluir e estão sempre sob consideração. A proposta deste trabalho foi fornecer ao leitor uma visão dos conceitos e procedimentos de validação utilizados no desenvolvimento de métodos instrumentais de separação pelos laboratórios industriais, acadêmicos e governamentais. Várias discussões foram inseridas no decorrer do texto, mas alguns pontos devem ser enfatizados para finalizar este tema.

As técnicas cromatográficas e eletroforéticas são alvos primordiais dos procedimentos de validação, pois envolvem monitoramentos que dizem respeito a aspectos como saúde pública, monitoramento ambiental, comércio exterior e controle de qualidade de produção.

A legislação, no que diz respeito à validação de metodologias, tem várias nuances e diferentes interpretações. Parte desta característica é intencional, pois permite a adaptação para cada tipo de problema. A legislação brasileira tem sido melhor definida nos últimos dois anos, através de resoluções e recomendações do INMETRO e ANVISA, inspiradas em diretrizes da ICH e do grupo EURACHEM. Para que um estudo de validação seja conduzido com sucesso, é necessário que se tenha amplo conhecimento da legislação referente às 
substâncias em estudo (pesticidas, fármacos, etc.) e das diretrizes propostas pelas agências reguladoras que atuam na(s) área(s) em questão. O ideal é que se faça uma opção por uma linha a ser seguida e que a utilize durante todo o processo.

Finalmente, é importante esclarecer que a validação de métodos deve ser planejada antes de seu desenvolvimento e execução. A estratégia de validação é específica e é influenciada pelo procedimento analítico utilizado, pela natureza e concentração do composto de interesse e pela matriz. Correlacionando-se o desenvolvimento, otimização e validação de métodos de uma maneira lógica e organizada, os laboratórios podem gerar resultados bastante eficientes e produtivos. A validação de métodos pode ser um processo tedioso, mas a qualidade dos resultados gerada está diretamente relacionada com a qualidade deste processo.

\section{REFERENCIAS}

1. Codex Alimentarius Commission on Methods of Analysis and Sampling; Criteria for Evaluating Acceptable Methods of Analysis for Codex Purposes, CX/MAS 95/3, 1995.

2. International Conference on Harmonisation (ICH); Validation of Analytical Procedures: Definitions and Terminology, Q2A (CPMP/ICH/381/95), 1995.

3. International Conference on Harmonisation (ICH); Validation of Analytical Procedures: Methodology, Q2B (CPMP/ICH/281/95), 1995.

4. Agência Nacional de Vigilância Sanitária (ANVISA); Resolução RE $n^{\circ} 899$, de 29/05/2003.

5. United States Food and Drug Administration (US-FDA); Guidance for Industry, Bioanalytical Method Validation, 2001.

6. United States Food and Drug Administration (US-FDA); Guidance for Industry, Analytical Procedures and Methods Validation, 2000.

7. United States Food and Drug Administration (US-FDA); Reviewer Guidance, Validation of Chromatographic Methods, 1994.

8. European Commission; Guidance Document on Residue Analytical Methods, SANCO/825/00, 2000.

9. Eurachem Working Group; The Fitness for Purpose of Analytical Methods, A Laboratory Guide to Method Validation and Related Topics, 1998.

10. Thompson, M.; Ellison, S. L. R.; Wood, R.; Pure Appl. Chem. 2002, 74, 835 .

11. Instituto Nacional de Metrologia, Normalização e Qualidade Industrial (INMETRO); Orientações sobre Validação de Métodos de Ensaios Químicos, DOQ-CGCRE-008, 2003.

12. Shabir, G. A.; J. Chromatogr., A 2003, 987, 57.

13. International Standard Organization; General Requirements for the Competence of Testing and Calibration Laboratories, ISO/IEC 17025, 1999.

14. United States Pharmacopeia Convention; US Pharmacopeia 24, Validation of Compendial Methods <1225>, Rockville, 1999.

15. World Health Organization Expert Committee on Specifications for Pharmaceutical Preparations; Thirty-second report, WHO Technical Report Series, No.823, Geneva, 1992.

16. Huber, L.; LC-GC Int. 1998, 11, 96.

17. Jenke, D. R.; Instrument. Sci. Technol. 1997, 25, 345.

18. Jenke, D. R.; Instrument. Sci. Technol. 1998, 26, 1.

19. Jenke, D. R.; Instrument. Sci. Technol. 1998, 26, 19.

20. Bruce, P.; Minkkinen, P.; Riekkola, M. L.; Mikrochim. Acta 1998, 128, 93.
21. Massart, D. L.; Smeyers-Verbeke, J.; Vandeginste, B.; Analusis 1994, 22, M14. 22. Swartz, M. E.; Krull, I. S.; Pharm. Technol. 1998, 2, 12.

23. Leite, F.; Validação em Análise Química, $4^{\mathrm{a}}$ ed., Editora Átomo: Campinas, 2002.

24. Snyder, L. R. ; Kirkland, J. J.; Glajch, J. L.; Practical HPLC Method Development, 2 ed., Wiley: New York, 1997, cap. 15.

25. Hill, A. R. C; Reynolds, S. L.; Analyst 1999, 124, 953.

26. van der Voet, H.; van Rhijn, J. A. H.; van de Wiel, H. J.; Anal. Chim. Acta 1999, 391, 159.

27. Horwitz, W.; Pure Appl. Chem. 1995, 67, 331.

28. Internacional Standard Organization; Precision of Test Methods, ISO 5725, 1994.

29. Krull, I.; Swartz, M.; LC-GC 1998, 16, 464.

30. United States Food and Drug Administration, Center for Drug Evaluation and Research (CDER); Review Guide: Validation of Chromatographic Methods, Rockville, 1993.

31. United States Food and Drug Administration, Center for Drug Evaluation and Research (CDER); General Principles of Validation, Rockville, 1987.

32. Vessman, J.; Stefan, R. I.; Staden, J. F. V.; Danzer, K.; Lindner, W.; Burns, D. T.; Fajgelj, A.; Müller, H.; Pure Appl. Chem. 2001, 73, 1381.

33. Augusto, F.; Andrade, J. C.; Custódio, R.; http://www.chemkeys.com, acessada em Maio 2004

34. Barros Neto, B.; Pimentel, M. F.; Araújo, M. C. U.; Quim. Nova 2002, 25, 856.

35. Custodio, R.; de Andrade, J. C.; Augusto, F.; Quim. Nova 1997, $20,219$.

36. Chui, Q. S. H.; Zucchini, R. R.; Lichtig, J.; Ouim. Nova 2001, 24, 374.

37. Barros Neto, B.; Scarminio, I. S.; Bruns, R. E.; Como Fazer Experimentos: Pesquisa e Desenvolvimento na Ciência e na Indústria, Editora da Unicamp: Campinas, 2001.

38. Green, J. M.; Anal. Chem. 1996, 68, A305.

39. Associação Grupo de Analistas de Resíduos de Pesticidas (GARP); Manual de Resíduos de Pesticidas em Alimentos (apostila), 1999.

40. Krull, I.; Swartz M.; LC-GC 1998, 16, 1084

41. Cuadros-Rodríguez, L.; Gámiz-Gracia, L.; Almansa-López, E. M; BosqueSendra, J. M.; Trends Anal. Chem. 2001, 20, 620.

42. Lanças, F. M.; Cromatografia em Fase Gasosa, Acta: São Carlos, 1993.

43. Egea-González, F. J.; Torres, M. E. H.; López, E. A.; Cuadros-Rodríguez, L.; Vidal, J. L. M.; J. Chromatogr., A 2002, 966, 155.

44. Cuadros-Rodríguez, L.; Garcia-Campaña, A. M.; Almansa-López, E. M; Egea-González, F. J.; Cano, M. L. C.; Frenich, A. G.; Martinez-Vidal, J. L.; Anal. Chim. Acta 2003, 478, 281.

45. Berg, R. G.; Murta, A. L. M.; Kugler, W.; Quim. Nova 1988, 11, 288.

46. Instituto Nacional de Metrologia, Normalização e Qualidade Industrial (INMETRO); Vocabulário Internacional de Termos Fundamentais e Gerais de Metrologia, $2^{\mathrm{a}}$ ed., 2000.

47. Vial, J.; Jardy, A.; Chromatographia 2001, 53, S-141.

48. Horwitz, W.; Kamps, L. R.; Boyer, K. W.; J. Assoc. Offic. Anal. Chem. 1980, 63, 1344.

49. International Standard Organization; Statistics-Vocabulary and SymbolsPart 1: Probability and General Statistical Terms, ISO 3534-1, 1993.

50. Thompson, M.; Ellison, S. L. R.; Fajgelj, A.; Willetts, P.; Wood, R.; Pure Appl. Chem. 1999, 71, 337.

51. Burns, D. T.; Danzer, K.; Townshend, A.; Pure Appl. Chem. 2002, 74, 2201.

52. de Andrade, J. C.; Quim. Nova 1987, 10, 159.

53. Miller, J. C.; Miller, J. N.; Statistics for Analytical Chemistry, $2^{\mathrm{a}}$ ed., Ellis Horwood: Chichester, 1988.

54. Box, G. E. P.; Hunter, W. G.; Hunter, J. S.; Statistics for Experimenters, Wiley: New York, 1987.

55. Heyden, Y. V.; Analusis 1994, 22, M27. 